\title{
ASYMPTOTICALLY OPTIMAL DESIGNS ON COMPACT ALGEBRAIC MANIFOLDS
}

\author{
UJUÉ ETAYO, JORDI MARZO AND JOAQUIM ORTEGA-CERDÀ
}

\begin{abstract}
We find $t$-designs on compact algebraic manifolds with a number of points comparable to the dimension of the space of polynomials of degree $t$ on the manifold. This generalizes results on the sphere by Bondarenko, Radchenko and Viazovska in [BRV13]. Of special interest is the particular case of the Grassmannians where our results improve the bounds that had been proved previously.
\end{abstract}

\section{INTRODUCTION}

Given a real affine algebraic manifold $\mathcal{M}$ endowed with the normalized Lebesgue measure $\mu_{\mathcal{M}}$, we say that a collection of $N$ points $x_{1}, \ldots, x_{N} \in \mathcal{M}$ is a $t$-design, also called averaging set or Chebyshev quadrature formula, if

$$
\int_{\mathcal{M}} P(x) d \mu_{\mathcal{M}}(x)=\frac{1}{N} \sum_{i=1}^{N} P\left(x_{i}\right),
$$

for all polynomials $P(x)$ of total degree less or equal than $t$.

The results for Chebyshev quadrature on the interval are classic, see for example the survey paper by Gautschi and the commentaries by Korevaar, [Gau14].

In the case of the sphere, these sets are known as spherical designs and were introduced by Delsarte, Goethals and Seidel [DGS77] in the context of algebraic combinatorics on spheres.

Since then, spherical designs have gained popularity in different areas of mathematics, ranging over geometry, algebraic and geometric combinatorics, and numerical analysis. See for instance the review [BB09] by Bannai and Bannai or the more recent review of Brauchart and Grabner [BG15].

Very early the notion of $t$-designs was considered in other contexts beyond the sphere. The projective designs were introduced by Hoggar [Hog82], who continued Neumaier's work in more general Delsarte spaces [Neu81]. They were further investigated by Lyubich and Shalatova, [LS04], as a tool to embed isometrically $\ell^{2}\left(\mathbb{R}^{n}\right)$ in $\ell^{2 t}\left(\mathbb{R}^{m}\right)$.

When the points in a $t$-designs are separated, they tend to be evenly distributed along the sphere or the projective space, and they are close to optimal for other problems, like minimization of Newtonian energy as observed in [HL08] and [BRV15].

Date: September 29, 2017.

This research has been partially supported by MTM2014-57590-P and MTM201451834-P grants by the Ministerio de Economía y Competitividad, Gobierno de España and by the Generalitat de Catalunya (project 2014 SGR 289). 
For this reason, in order to have evenly distributed subspaces, the $t$ designs on Grassmannians were considered by Bachoc, Coulangeon and Nebe in [BCN02]. In [BEG16] Breger, Ehler and Gräf make some numerical study of approximation problems in Grassmannians and observe that two possible notions of designs in Grassmannians (one of them using polynomials as we did and another when one replaces polynomials by eigenvectors of the Laplacian as in [BCN02]) are indeed related.

The existence of designs in a very general setting was proved by Seymour and Zaslavsky in [SZ84], see also [AdR88]. One challenging problem is to find designs with as few points as possible.

In [Kan15] Kane studied the existence of designs in path-connected spaces. In particular for the Grassmannian $G\left(k, \mathbb{R}^{n}\right)$, linear subspaces of $\mathbb{R}^{n}$ of dimension $k$, he proved that one can find $t$-designs with a number of points of order $O\left(t^{2 k(n k)}\right)$ and he conjectured that it should be of order $O\left(t^{k(n k)}\right)$ since this is the dimension of the corresponding space of polynomials.

A breakthrough on the existence of designs of size of optimal asymptotic order was obtained in [BRV13], where the conjecture by Korevaar and Meyers was settled, [KM93, KM94]. The conjecture said that there are $t$-designs with $O\left(t^{d}\right)$ points in the $d$-dimensional sphere. This is asymptotically the best rate possible. The proof is obtained by a fixed point theorem, thus it is not constructive. On the other hand their method is very flexible, so they could improve it in [BRV15] to obtain separated spherical designs with the same cardinality.

As a further evidence of the flexibility of their method we are going to adapt it to the setting of a general algebraic manifold and obtain sharp estimates on the number of points needed for a $t$-design. The two main ingredients that are needed to adapt their method to this setting are the construction of area regular partitions in manifolds (this has been recently proved by Gigante and Leopardi in [GL15]) and the existence of a sampling-type inequalities (Marcinkiewicz-Zygmund inequalities) for polynomials. This last ingredient essentially follows from a Bernstein-type inequality for polynomials in algebraic varieties proved in [BOC15]. If we specialize our results we confirm the conjecture for Grassmannians stated in [Kan15], and we also find the right order of quadrature formulas on ellipses, as suggested in [KM94, Remark 3.2].

\section{Definitions and Main Results}

Let $\mathcal{M}$ be a smooth, connected and compact affine algebraic manifold of dimension $d$ in $\mathbb{R}^{n}$

$$
\mathcal{M}=\left\{x \in \mathbb{R}^{n}: p_{1}(x)=\cdots=p_{r}(x)=0\right\},
$$

where $p_{1}, \ldots, p_{r} \in \mathbb{R}[X]$ are polynomials with real coefficients and the normal space at $x \in \mathcal{M}$ is of dimension $n-d$. This normal space is generated by $\nabla p_{1}(x), \ldots, \nabla p_{r}(x)$, where $\nabla p$ denotes the gradient of the polynomial $p$ in $\mathbb{R}^{n}$. We consider in $\mathcal{M}$ the $d$-dimensional Hausdorff measure (i.e. the Lebesgue measure) $\mu_{\mathcal{M}}$, normalized by $\mu_{\mathcal{M}}(\mathcal{M})=1$. We denote as $d(x, y)$ the geodesic distance between $x, y \in \mathcal{M}$. 
Let $X \subset \mathbb{C}^{n}$ be the complexification of $\mathcal{M}$, i.e. the complex zero set of the real polynomials $p_{1}(x), \ldots, p_{r}(x)$. The Lebesgue measure in $X$ will be denoted by $\mu_{X}$.

The space of real algebraic polynomials on $\mathcal{M}$ of total degree at most $t$, denoted by $\mathcal{P}_{t}=\mathcal{P}_{t}(\mathcal{M})$ is the restriction to $\mathcal{M}$ of the space of real polynomials in $n$ variables. The dimension of the space $\mathcal{P}_{t}(\mathcal{M})$ is given by the Hilbert polynomial and it satisfies:

$$
\operatorname{dim} \mathcal{P}_{t}(\mathcal{M})=\operatorname{deg}(\mathcal{M}) t^{d}+O\left(t^{d-1}\right) .
$$

Let $\mathcal{P}_{t}^{0}$ be the Hilbert space of polynomials in $\mathcal{P}_{t}$ with zero mean

$$
\int_{\mathcal{M}} P(x) d \mu_{\mathcal{M}}(x)=0
$$

with respect to the usual inner product

$$
\langle P, Q\rangle=\int_{\mathcal{M}} P(x) Q(x) d \mu_{\mathcal{M}}(x) .
$$

$\mathcal{P}_{t}^{0}$ has a reproducing kernel i.e. for each $x \in \mathcal{M}$ there exists a unique polynomial $K_{x} \in \mathcal{P}_{t}^{0}$ such that

$$
\left\langle Q, K_{x}\right\rangle=Q(x)
$$

for all $Q \in \mathcal{P}_{t}^{0}$.

It is clear that $x_{1}, \ldots, x_{N} \in \mathcal{M}$ is a $t$-design if and only if

$$
\sum_{i=1}^{N} K_{x_{i}}=0 .
$$

The existence of designs was already proved in a very general setting in [SZ84], our aim is to show, adapting the techniques from [BRV13] that one can reach the right order given by the following result, see also [dlHP05].

Proposition 2.1. If $x_{1}, \ldots, x_{N} \in \mathcal{M}$ is a t-design in $\mathcal{M}$, then $N \gtrsim t^{d}$.

Proof. Let $s$ be such that $2 s=t$, if $t$ is even, and such that $2 s+1=t$ otherwise. We have that

$$
\int_{\mathcal{M}} P^{2}(x) d \mu_{\mathcal{M}}(x)=\frac{1}{N} \sum_{i=1}^{N} P^{2}\left(x_{i}\right), \text { for } P \in \mathcal{P}_{s} .
$$

Suppose that $N<\operatorname{dim} \mathcal{P}_{s}$. Then for $P_{1}, \ldots, P_{N} \in \mathcal{P}_{s}$ linearly independent we have that

$$
\operatorname{det}\left(P_{j}\left(x_{i}\right)\right)_{i, j=1, \ldots, N} \neq 0 .
$$

Indeed, if the determinant above vanishes, there exist a non-trivial linear combination

$$
\sum_{j=1}^{N} \alpha_{j} P_{j}\left(x_{i}\right)=0, \quad i=1, \ldots, N,
$$

and we get a contradiction from

$$
\int_{\mathcal{M}}\left(\sum_{j=1}^{N} \alpha_{j} P_{j}(x)\right)^{2} d \mu_{\mathcal{M}}(x)=0 .
$$


As $\operatorname{det}\left(P_{j}\left(x_{i}\right)\right)_{i, j=1, \ldots, N} \neq 0$, there exist $Q_{1}, \ldots, Q_{N} \in \mathcal{P}_{s}$ such that $Q_{j}\left(x_{i}\right)=$ $\delta_{i j}$, for $i, j=1, \ldots, N$, and $Q \in \operatorname{span}\left\{Q_{1}, \ldots, Q_{N}\right\}^{\perp}$. Then

$$
0=\int_{\mathcal{M}} Q(x) Q_{j}(x) d \mu_{\mathcal{M}}(x)=\frac{1}{N} Q\left(x_{j}\right), \quad j=1, \ldots, N
$$

and from $\int_{\mathcal{M}} Q(x)^{2} d \mu_{\mathcal{M}}(x)=0$ we get that $Q(x)=0$.

Remark. If $x_{1}, \ldots, x_{N} \in \mathcal{M}$ is a $t$-design in $\mathcal{M}$, for even $t$, and $N=$ $\operatorname{dim} \mathcal{P}_{t / 2}(\mathcal{M})$, it is easy to see that the set of reproducing kernels of the space $\mathcal{P}_{t / 2}(\mathcal{M})$ on those points

$$
K_{t / 2}\left(\cdot, x_{j}\right), j=1, \ldots N
$$

form an orthogonal basis of $\mathcal{P}_{t / 2}(\mathcal{M})$ and

$$
K_{t / 2}\left(x_{j}, x_{j}\right)=\left\|K_{t / 2}\left(\cdot, x_{j}\right)\right\|_{L^{2}(\mathcal{M})}^{2}=N,
$$

for all $j=1, \ldots, N$. The existence (or not) of these, so-called, tight designs in a variety $\mathcal{M}$ seems to be a difficult problem.

In the case of the sphere $\mathbb{S}^{d}$ the (sharp) lower bounds tell us that if $x_{1}, \ldots, x_{N} \in \mathbb{S}^{d}$ is a $t$-design

$$
N \geq\left(\begin{array}{c}
d+s \\
d
\end{array}\right)+\left(\begin{array}{c}
d+s-1 \\
d
\end{array}\right)=\operatorname{dim} \mathcal{P}_{s}\left(\mathbb{S}^{d}\right), \quad N \geq 2\left(\begin{array}{c}
d+s \\
d
\end{array}\right)
$$

for $t=2 s$ and $t=2 s+1$, respectively. For the sphere $\mathbb{S}^{d}$ there are a few tight spherical designs, see [BD79,BD80], for which these lower bounds are attained. The tight spherical designs with larger cardinality are the kissing tight 4-design for $\mathbb{S}^{21}$ of $275=\operatorname{dim} \mathcal{P}_{2}\left(\mathbb{S}^{21}\right)$ points for even $t$, and the 11-design for $\mathbb{S}^{23}$ of 196560 points from the Leech lattice for odd $t$.

Our main result is the following theorem where we show the existence of designs with cardinality $N$ for all $N \gtrsim \operatorname{dim} \mathcal{P}_{t}(\mathcal{M})$.

Theorem 2.2. There is a constant $C_{\mathcal{M}}$ depending only on $\mathcal{M}$ such that for each $N \geq C_{\mathcal{M}} t^{d}$ there are $t$-designs in $\mathcal{M}$ with $N$ points.

Besides the sharp result for the sphere in [BRV13], Kuijlaars has proved on the torus on $\mathbb{R}^{3}$ the existence of Chebyshev quadratures with $C t^{2}$ points for polynomials of degree $t$, [Kui95]. In [Kan15] the author obtained results in the very general setting of path-connected topological spaces. His result in our setting provides designs for any $N \gtrsim t^{2 d}$.

Observe that a sort of converse to Theorem 2.2 also holds. Indeed, let $\mathcal{M} \subset \mathbb{R}^{n}$ be a smooth compact real manifold of dimension $d$. The existence of a $t$-design of order $t^{d}$ in $\mathcal{M}$ implies that the dimension the space of polynomials in $n$ variables of degree at most $t / 2$, restricted to $\mathcal{M}$, is of order $t^{d}$. This implies that $\mathcal{M}$ is cut out by a system of polynomial equations.

To prove Theorem 2.2, we follow the strategy of [BRV13]. The main ingredients are a result from Brouwer degree theory and MarcinkiewiczZygmund inequalities for spaces of polynomials $\mathcal{P}_{t}(\mathcal{M})$. In [BRV13] the authors borrow the Marcinkiewicz-Zygmund inequalities on the sphere from [MNW01, Theorem 3.1], see also [DX13, Theorem 6.4.4]. We will prove the analogue for algebraic polynomials on algebraic varieties.

To state our results we have to define area regular partitions. 
A finite family of closed sets $R_{1}, \ldots, R_{N} \subset \mathcal{M}$ is an area regular partition of $\mathcal{M}$ if

$$
\mu_{\mathcal{M}}\left(R_{i}\right)=1 / N, \bigcup_{i=1}^{N} R_{i}=\mathcal{M}, \text { and } \mu_{\mathcal{M}}\left(R_{i} \cap R_{j}\right)=0 \text { for } i \neq j .
$$

The diameter of the partition $\mathcal{R}=\left\{R_{1}, \ldots, R_{N}\right\}$ is

$$
\|\mathcal{R}\|=\max _{i=1, \ldots, N} \max _{x, y \in R_{i}} d(x, y) .
$$

Following previous constructions for the sphere, it is not difficult to deduce the existence of area regular partitions with diameter comparable to $N^{-1 / d}$ for any compact algebraic variety, see for example [RSZ94] and the references therein. The existence of such a partition in our case can be deduced also from a recent result by Gigante and Leopardi for Ahlfors regular metric measure spaces, see [GL15, Theorem 2].

Proposition 2.3. For any $N \geq N_{0}=N_{0}(\mathcal{M}) \in \mathbb{N}$ there exists an area regular partition $\mathcal{R}=\left\{R_{1}, \ldots, R_{N}\right\}$ of $\mathcal{M}$, with $\mu_{\mathcal{M}}\left(R_{i}\right)=1 / N$ for all $1 \leq i \leq N$, and such that

$$
B\left(c_{1} N^{-1 / d}\right) \subset R_{i} \subset B\left(c_{2} N^{-1 / d}\right),
$$

where $B(r)$ is a geodesic ball in $\mathcal{M}$ of radius $r>0$ and the constants $c_{1}, c_{2}$ depend only on $\mathcal{M}$.

Our result about Marcinkiewicz-Zygmund inequalities is the following:

Theorem 2.4. There exists a constant $A=A(\mathcal{M})>0$ such that if $N \geq A t^{d}$ and $\mathcal{R}=\left\{R_{1}, \ldots, R_{N}\right\}$ is an area regular partition of $\mathcal{M}$ as in (2). Then for all $P \in \mathcal{P}_{t}$

$$
\frac{1}{2} \int_{\mathcal{M}}|P(x)| d \mu_{\mathcal{M}}(x) \leq \frac{1}{N} \sum_{i=1}^{N}\left|P\left(x_{i}\right)\right| \leq \frac{3}{2} \int_{\mathcal{M}}|P(x)| d \mu_{\mathcal{M}}(x),
$$

for any choice of $x_{i} \in R_{i}$, with $1 \leq i \leq N$.

We will need also Marcinkiewicz-Zygmund inequalities for tangential gradients of polynomials. Observe that, unlike for the sphere, for a general variety the tangential gradient is not necessarily a polynomial.

Definition 2.5. Given a differentiable function $f$ in $\mathcal{M} \subset \mathbb{R}^{n}$. The tangential gradient of $f$ at a point $x \in \mathcal{M}$, denoted as $\nabla_{\mathcal{M}} f(x)$ is the orthogonal projection of the gradient $\nabla f(x)$ onto the tangent space of $\mathcal{M}$ at $x$.

Corollary 2.6. There exists a constant $A=A(\mathcal{M})>0$ such that if $N \geq$ $A t^{d}$ and $\mathcal{R}=\left\{R_{1}, \ldots, R_{N}\right\}$ is an area regular partition of $\mathcal{M}$ as in (2).

Then for all $P \in \mathcal{P}_{t}$

$\frac{1}{K_{\mathcal{M}}} \int_{\mathcal{M}}\left|\nabla_{\mathcal{M}} P(x)\right| d \mu_{\mathcal{M}}(x) \leq \frac{1}{N} \sum_{i=1}^{N}\left|\nabla_{\mathcal{M}} P\left(x_{i}\right)\right| \leq K_{\mathcal{M}} \int_{\mathcal{M}}\left|\nabla_{\mathcal{M}} P(x)\right| d \mu_{\mathcal{M}}(x)$,

where $K_{\mathcal{M}}=3 \sqrt{d}\left(\begin{array}{c}r \\ n-d\end{array}\right) C_{\mathcal{M}}$ for $C_{\mathcal{M}}>0$ depending only on $\mathcal{M}$ and for any choice of $x_{i} \in R_{i}$, with $1 \leq i \leq N$. 
As in [BRV13], the last ingredient of the proof of Theorem 2.2 is the following result from Brouwer degree theory:

Theorem 2.7 ([CC06, Theorem 1.2.9]). Let $f: \mathbb{R}^{n} \longrightarrow \mathbb{R}^{n}$ be a continuous mapping and $\Omega$ an open bounded subset, with boundary $\partial \Omega$, such that $0 \in$ $\Omega \subset \mathbb{R}^{n}$. If $\langle x, f(x)\rangle>0$ for all $x \in \partial \Omega$, then there exists $x \in \Omega$ satisfying $f(x)=0$.

Defining the convenient mapping from $\mathcal{P}_{t}$ into itself, this result will give us (1).

\section{Proofs}

First we prove the Marcinkiewicz-Zygmund inequalities in the algebraic variety $\mathcal{M}$ (Theorem 2.4). Similar results have been obtained also in general compact Riemannian manifolds for spaces of, so-called, diffusion polynomials (i.e. eigenfunctions of elliptic differential operators, in particular, for the Laplace-Beltrami operator), [FM10,FM11]. In the proof we use the following result from Berman and Ortega-Cerdà, analogous to the Plancherel-Polya inequality for entire functions of exponential type, [You01].

Lemma 3.1 ([BOC15, Theorem 10]). There exists a constant $C=C_{\mathcal{M}}>0$ such that for all polynomials $P \in \mathcal{P}_{t}$ the following inequality holds

$$
\int_{U\left(\frac{1}{t}\right)}|P(x)| d \mu_{X}(x) \leq \frac{C}{t^{d}} \int_{\mathcal{M}}|P(x)| d \mu_{\mathcal{M}}(x),
$$

where $U\left(\frac{1}{t}\right)=\left\{x \in X: d(x, \mathcal{M}) \leq \frac{1}{t}\right\}$.

Proof of Theorem 2.4. During the proof, we will denote by $C$ any constant depending on $\mathcal{M}$.

Given the degree $t \in \mathbb{N}$, let $N=t^{d} / a^{d} \geq A t^{d}$, where $A=A(\mathcal{M})>0$ is some constant to be determined. Consider the area regular partition with $N$ points given by Proposition 2.3. By assumption the balls satisfy

$$
B\left(a c_{1} t^{-1}\right) \subset R_{i} \subset B\left(a c_{2} t^{-1}\right),
$$

and therefore the diameter of $\mathcal{R}$ satisfies

$$
\frac{2 a c_{1}}{t} \leq\|\mathcal{R}\| \leq \frac{2 a c_{2}}{t} .
$$

Then we compare the average of $P$ and the integral and we obtain:

$$
\begin{aligned}
\left|\frac{1}{N} \sum_{i=1}^{N}\right| P\left(x_{i}\right)\left|-\int_{\mathcal{M}}\right| P(x)\left|d \mu_{\mathcal{M}}(x)\right| & \leq \sum_{i=1}^{N}\left|\int_{R_{i}}\right| P\left(x_{i}\right)|-| P(x)\left|d \mu_{\mathcal{M}}(x)\right| \\
& \leq \frac{\|\mathcal{R}\|}{N} \sum_{i=1}^{N}\left|\nabla_{\mathcal{M}} P\left(x_{i}^{\prime}\right)\right|,
\end{aligned}
$$

where $x_{i}^{\prime}$ is such that $\left|\nabla_{\mathcal{M}} P\left(x_{i}^{\prime}\right)\right| \geq\left|\nabla_{\mathcal{M}} P(x)\right|$ for all $x \in R_{i}$. Observe that we can take $x_{i}^{\prime}$ in the ball $B\left(a c_{2} t^{-1}\right)$ containing $R_{i}$. 
Consider now each $x_{i}^{\prime}$ as a point of $X$, the complex variety, and apply Cauchy's inequality

$$
\left|\nabla_{\mathcal{M}} P\left(x_{i}^{\prime}\right)\right| \leq \frac{C}{\left(\frac{1}{t}\right)^{2 d+1}} \int_{B_{X}\left(x_{i}^{\prime}, \frac{1}{t}\right)}|P(z)| d \mu_{X}(z),
$$

where $C$ is a constant depending on $\mathcal{M}$ and $B_{X}\left(x_{i}^{\prime}, t^{-1}\right)$ is a ball in $X$.

Observe that, for $i=1, \ldots, N$, the number of balls $B_{X}\left(x_{i}^{\prime}, t^{-1}\right)$ with nonempty intersection is bounded independently of $t$. Indeed, if $I \subset\{1, \ldots, N\}$ there exist $C$ depending only on $\mathcal{M}$ such that if

$$
\bigcap_{j \in I} B_{X}\left(x_{j}^{\prime}, t^{-1}\right) \neq \emptyset \text {, then } \bigcap_{j \in I} B\left(x_{j}^{\prime}, C t^{-1}\right) \neq \emptyset,
$$

and $R_{j} \subset B\left(x_{j}^{\prime}, C t^{-1}\right)$ for $j \in I$. As each $R_{j}$ contains a (disjoint) ball of radius $a c_{1} t^{-1}$, we get that

$$
\operatorname{card}(I) a^{d} \leq C .
$$

We can bound the sum on (5) by the sum of integrals on the corresponding balls and using the bounded intersections we can pass to a tubular domain around $\mathcal{M}$ defined as in Lemma 3.1

$$
\sum_{i=1}^{N} \int_{B_{X}\left(x_{i}^{\prime}, \frac{1}{t}\right)}|P(z)| d \mu_{X}(z) \leq \frac{C}{a^{d}} \int_{U\left(\frac{1}{t}\right)}|P(z)| d \mu_{X}(z) .
$$

Finally we apply Lemma 3.1 and we get that (5) is bounded by

$$
\frac{\|\mathcal{R}\|}{N} \sum_{i=1}^{N}\left|\nabla_{\mathcal{M}} P\left(x_{i}^{\prime}\right)\right| \leq C \frac{\|\mathcal{R}\| t^{d+1}}{a^{d} N} \int_{\mathcal{M}}|P(x)| d \mu_{\mathcal{M}}(x) .
$$

By using that $N=t^{d} / a^{d}$ and the upper bound for $\|\mathcal{R}\|$ it is clearly enough to take $A=\left(4 C c_{2}\right)^{d}$.

To prove the Marcinkiewicz-Zygmund inequalities for the tangential gradient (Corollary 2.6) we use the following inequality for vectors of polynomials.

Corollary 3.2. Let $k \in \mathbb{N}$ be a fixed constant. There exists a constant $A=A(\mathcal{M}, k)>0$ such that if $\mathcal{R}=\left\{R_{1}, \ldots, R_{N}\right\}$ is an area regular partition as in (2) with $N \geq A t^{d}$, then a vectorial Marcinkiewicz-Zygmund inequality for polynomials of degree at most $t+k$ holds. That is, for all vectors of polynomials $Q(x)=\left(Q_{1}(x), \ldots, Q_{m}(x)\right)$ with $m \leq 2 d$ and $Q_{j}(x) \in \mathcal{P}_{t+k}(\mathcal{M})$ we have that

(6) $\quad \frac{1}{3 \sqrt{d}} \int_{\mathcal{M}}|Q(x)| d \mu_{\mathcal{M}}(x) \leq \frac{1}{N} \sum_{i=1}^{N}\left|Q\left(x_{i}\right)\right| \leq 3 \sqrt{d} \int_{\mathcal{M}}|Q(x)| d \mu_{\mathcal{M}}(x)$,

for any election of $x_{i} \in R_{i}$.

Proof. Let $A$ be the constant given by the Theorem 2.4 when we replace $t+k$ with $t$. Then we use that

$$
|Q(x)| \leq \sum_{j=1}^{m}\left|Q_{j}(x)\right| \leq \sqrt{m}|Q(x)|,
$$

and we apply the previous result for each $Q_{j}(x)$. 
In [BRV13] this result above was enough because the tangential gradient on the sphere of a spherical polynomial can be written as a vector of spherical polynomials (i.e. polynomials restricted to the sphere). This does no longer hold in our case and we have to be more careful.

Proof of Corollary 2.6. Let $\mathcal{M}$ be given as the common zero set of the real polynomials $p_{1}(x), \ldots, p_{r}(x)$.

Since $\mathcal{M}$ is smooth of dimension $d$, for all $x \in \mathcal{M}$ the normal space to $\mathcal{M}$ on $x$ is generated by

$$
\nabla p_{i_{1}}(x), \ldots, \nabla p_{i_{n-d}}(x),
$$

where the index $i_{1}<\cdots<i_{n-d}$ (which may depend on $x$ ) is a subset of $\{1, \ldots, r\}$.

Assume that $i_{j}=j$ for $j=1, \ldots, n-d$. By the Gram-Schmidt determinanttype formula we obtain an orthogonal basis $u_{1}(x), \ldots, u_{n-d}(x)$ of the normal space at $x$ by the following determinants

$$
u_{i}(x)=\left|\begin{array}{cccc}
\left\langle\nabla p_{1}(x), \nabla p_{1}(x)\right\rangle & \left\langle\nabla p_{2}(x), \nabla p_{1}(x)\right\rangle & \ldots & \left\langle\nabla p_{i}(x), \nabla p_{1}(x)\right\rangle \\
\left\langle\nabla p_{1}(x), \nabla p_{2}(x)\right\rangle & \left\langle\nabla p_{2}(x), \nabla p_{2}(x)\right\rangle & \ldots & \left\langle\nabla p_{i}(x), \nabla p_{2}(x)\right\rangle \\
\vdots & \vdots & \ddots & \vdots \\
\left\langle\nabla p_{1}(x), \nabla p_{i-1}(x)\right\rangle & \left\langle\nabla p_{2}(x), \nabla p_{i-1}(x)\right\rangle & \ldots & \left\langle\nabla p_{i}(x), \nabla p_{i-1}(x)\right\rangle \\
\nabla p_{1}(x) & \nabla p_{2}(x) & \ldots & \nabla p_{i}(x)
\end{array}\right| .
$$

Observe that it is a formal determinant that must be computed developing by the last row. Since every $\nabla p_{i}(x)$ is a vector of polynomials, the product $\left\langle\nabla p_{i}(x), \nabla p_{j}(x)\right\rangle$ is also a polynomial and therefore $u_{i}(x)$ is also a vector of polynomials of total degree bounded by a constant depending only on $\mathcal{M}$. The tangential gradient of $P$ at $x \in \mathcal{M}$ is then

$$
\nabla_{\mathcal{M}} P(x)=\nabla P(x)-\sum_{i=1}^{n-d} \frac{\left\langle\nabla P(x), u_{i}(x)\right\rangle u_{i}(x)}{\left|u_{i}(x)\right|^{2}} .
$$

If there are $n-d$ polynomials defining the normal space to $\mathcal{M}$ in all the variety, in particular, for the sphere or any other algebraic hypersurface, the result follows because one can apply Corollary 3.2 to the vector of polynomials

$$
\left(\prod_{i=1}^{n-d}\left|u_{i}(x)\right|^{2}\right) \nabla_{\mathcal{M}} P(x)
$$

and use that as $\mathcal{M}$ is smooth

$$
0<C_{\mathcal{M}}^{-1} \leq \prod_{i=1}^{n-d}\left|u_{i}(x)\right|^{2} \leq C_{\mathcal{M}}
$$

for some $C_{\mathcal{M}}>0$.

Now for any $I \subset\{1, \ldots, r\}$ with $|I|=n-d$ we can define the vectors of polynomials $u_{j}^{I}(x)$ for $j=1, \ldots, n-d$ (where maybe some of the polynomials are zero) and by Corollary 3.2 the Marcinkiewicz-Zygmund inequalities hold for

$$
\left(\prod_{i=1}^{n-d}\left|u_{i}^{I}(x)\right|^{2}\right) \nabla P(x)-\sum_{i=1}^{n-d}\left\langle\nabla P(x), u_{i}^{I}(x)\right\rangle u_{i}^{I}(x) \prod_{j \neq i}\left|u_{j}^{I}(x)\right|^{2} .
$$


Clearly, Marcinkiewicz-Zygmund inequalities hold also taking supremum for the subsets $I \subset\{1, \ldots, r\}$ with $|I|=n-d$.

Indeed, now as

$$
C_{\mathcal{M}}^{-1}\left|\nabla_{\mathcal{M}} P(x)\right| \leq \sup _{\substack{I \subset\{1, \ldots, r\} \\|I|=n-d}} \prod_{i=1}^{n-d}\left|u_{i}^{I}(x)\right|^{2}\left|\nabla_{\mathcal{M}} P(x)\right| \leq C_{\mathcal{M}}\left|\nabla_{\mathcal{M}} P(x)\right|,
$$

for some constant $C_{\mathcal{M}}>0$, the result follows because for $v_{I}(x)$ as in (8)

$$
\left(\begin{array}{c}
r \\
n-d
\end{array}\right)^{-1} \sum_{\substack{I \subset\{1, \ldots, r\} \\
|I|=n-d}}\left|v_{I}(x)\right| \leq \sup _{\substack{I \subset\{1, \ldots, r\} \\
|I|=n-d}}\left|v_{I}(x)\right| \leq \sum_{\substack{I \subset\{1, \ldots, r\} \\
|I|=n-d}}\left|v_{I}(x)\right|,
$$

for all $x \in \mathcal{M}$.

The following result is the main tool to define a mapping with the right properties and apply Theorem 2.7.

Lemma 3.3. There exists a constant $A=A(\mathcal{M})>0$ such that if $N \geq A t^{d}$ then there exists a continuous mapping

$$
\begin{aligned}
\mathcal{P}_{t}^{0} & \rightarrow \mathcal{M}^{N} \\
P & \mapsto\left(x_{1}(P), \ldots, x_{N}(P)\right),
\end{aligned}
$$

such that for all $P \in \mathcal{P}_{t}^{0}$ with $\int_{\mathcal{M}}\left|\nabla_{\mathcal{M}} P(x)\right| d \mu(x)=1$

$$
\left\langle P, \sum_{i=1}^{N} K_{x_{i}(P)}\right\rangle=\sum_{i=1}^{N} P\left(x_{i}(P)\right)>0 .
$$

Proof. Let $A=A(\mathcal{M})>0$ be given by Corollary 2.6. Let $N \geq A t^{d}$ and $\mathcal{R}=\left\{R_{1}, \ldots, R_{N}\right\}$ be an area regular partition of $\mathcal{M}$ as in (2).

Given a polynomial $P$, we define in $\mathcal{M}$ the vector field $X_{P}=\nabla_{\mathcal{M}} P / U_{\epsilon}\left(\left|\nabla_{\mathcal{M}} P\right|\right)$, where $U_{\epsilon}: \mathbb{R}^{+} \rightarrow \mathbb{R}$ is a smooth increasing function such that $U_{\epsilon}(x)=\epsilon / 2$ if $0 \leq x \leq \varepsilon / 2$ and $U_{\epsilon}(x)=x$ if $x \geq \varepsilon$ for some $\epsilon$ fixed. Since $U_{\epsilon}(x)$ is smooth, the vector field $X_{P}$ is smooth on $\mathcal{M}$ and depends continuously on $P$.

Now for each $1 \leq i \leq N$ we consider the map $y_{i}:[0, \infty) \longrightarrow \mathcal{M}$ that satisfies the differential equation

$$
\left\{\begin{array}{l}
\frac{\partial}{\partial s} y_{i}(s)=X_{P}\left(y_{i}(s)\right) \\
y_{i}(0)=x_{i}
\end{array}\right.
$$

where $x_{i} \in R_{i}$. The differential equation changes for each $P \in \mathcal{P}$, thus we will sometimes denote $y_{i}(s)$ as $y_{i}(P, s)$ to stress the dependence on $P$.

Note that the quantity $\sum_{i=1}^{N} P\left(x_{i}\right)$ is small since $\int_{\mathcal{M}} P(x) d \mu(x)=0$. In order to increase this quantity, we move from the point $x_{i}$ in the direction that increases $P\left(x_{i}\right)$, that is, the direction given by the vector $\nabla_{\mathcal{M}} P\left(x_{i}\right)$.

Since the vector field $X_{P}$ is smooth, each $y_{i}$ is well defined and continuous in both $P$ and $s$. For a fixed $s_{0}>0$ to be determined, define the continuous mapping

$$
\mathcal{P}_{t}^{0} \ni P \mapsto\left(x_{1}(P), \ldots, x_{N}(P)\right)=\left(y_{1}\left(P, s_{0}\right), \ldots, y_{N}\left(P, s_{0}\right)\right) .
$$


Now, following [BRV13] we split

$$
\begin{aligned}
\frac{1}{N} \sum_{i=1}^{N} P\left(x_{i}(P)\right) & =\frac{1}{N} \sum_{i=1}^{N} P\left(x_{i}\right)+\int_{0}^{s_{0}} \frac{d}{d s}\left[\frac{1}{N} \sum_{i=1}^{N} P\left(y_{i}(P, s)\right)\right] d s \\
& \geq \int_{0}^{s_{0}} \frac{d}{d s}\left[\frac{1}{N} \sum_{i=1}^{N} P\left(y_{i}(P, s)\right)\right] d s-\left|\frac{1}{N} \sum_{i=1}^{N} P\left(x_{i}\right)\right| .
\end{aligned}
$$

We can modify the area regular partition without losing its essential properties. Indeed, if $x_{i}^{\prime}$ belongs to a ball $B\left(C_{\mathcal{M}}\|\mathcal{R}\|\right)$ containing $R_{i}$, where $C_{\mathcal{M}}>0$ is a constant depending only on $\mathcal{M}$ then, defining

$$
R_{i}^{\prime}=\left(R_{i} \backslash \cup_{j=1}^{N}\left\{x_{j}^{\prime}\right\}\right) \cup\left\{x_{i}^{\prime}\right\},
$$

we get an area regular partition with the same properties, i.e.

$$
B\left(c_{1}^{\prime} N^{-1 / d}\right) \subset R_{i}^{\prime} \subset B\left(c_{2}^{\prime} N^{-1 / d}\right),
$$

for some constants $c_{1}^{\prime}, c_{2}^{\prime}$ depending only on $\mathcal{M}$.

As in (5) and using that $P \in \mathcal{P}_{t}^{0}$ has mean zero, we get

$$
\left|\frac{1}{N} \sum_{i=1}^{N} P\left(x_{i}\right)\right| \leq \frac{\|\mathcal{R}\|}{N} \sum_{i=1}^{N}\left|\nabla_{\mathcal{M}} P\left(x_{i}^{\prime}\right)\right|,
$$

where $x_{i}^{\prime}$ is a point in the ball $B\left(c_{2}^{\prime} N^{-1 / d}\right)$ containing $R_{i}$ where $\left|\nabla_{\mathcal{M}} P(x)\right|$ attains its maximum. Applying the the right-hand side inequality in (4) and the modification of the area regular partition mentioned above we get that

$$
\left|\frac{1}{N} \sum_{i=1}^{N} P\left(x_{i}\right)\right| \leq K_{\mathcal{M}}\|\mathcal{R}\| .
$$

For any fixed $0<s<C_{\mathcal{M}}\|\mathcal{R}\|$ we apply the left-hand side inequality in (4) and again a modification of the area regular partition to get

$$
\begin{aligned}
& \frac{d}{d s}\left[\frac{1}{N} \sum_{i=1}^{N} P\left(y_{i}(P, s)\right)\right]=\frac{1}{N} \sum_{i=1}^{N} \frac{\left|\nabla_{\mathcal{M}} P\left(y_{i}(P, s)\right)\right|^{2}}{U_{\epsilon}\left(\left|\nabla_{\mathcal{M}} P\left(y_{i}(P, s)\right)\right|\right)} \\
& \geq \frac{1}{N} \sum_{i:\left|\nabla_{\mathcal{M}} P\left(y_{i}(P, s)\right)\right| \geq \epsilon}\left|\nabla_{\mathcal{M}} P\left(y_{i}(P, s)\right)\right| \geq \frac{1}{N} \sum_{i=1}^{N}\left|\nabla_{\mathcal{M}} P\left(y_{i}(P, s)\right)\right|-\epsilon \\
& \geq \frac{1}{K_{\mathcal{M}}}-\epsilon .
\end{aligned}
$$

So, finally taking $s_{0}=3 K_{\mathcal{M}}^{2}\|\mathcal{R}\|$ and $\epsilon=\frac{1}{2 K_{\mathcal{M}}}$ we get from (11)

$$
\frac{1}{N} \sum_{i=1}^{N} P\left(x_{i}(P)\right) \geq \frac{K_{\mathcal{M}}\|\mathcal{R}\|}{2}>0 .
$$

Proof of Theorem 2.2. Fix $t$ and define

$$
\Omega=\left\{P \in \mathcal{P}_{t}^{0}: \int_{\mathcal{M}}\left|\nabla_{\mathcal{M}} P(x)\right| d \mu_{\mathcal{M}}(x)<1\right\}
$$


which is clearly an open, bounded subset of $\mathcal{P}_{t}^{0}$ such that $0 \in \Omega \subset \mathcal{P}_{t}^{0}$. Take $N \geq A t^{d}$ for $A$ given by Corollary 2.6 and let $x_{i}(P)$ be the points defined for $P \in \partial \Omega$.

From the continuity of $P \mapsto\left(x_{1}(P), \ldots, x_{N}(P)\right)$ it follows that

$$
\mathcal{P}_{t}^{0} \ni P \mapsto \sum_{i=1}^{N} K_{x_{i}(P)},
$$

is continuous and from Lemma 3.3, for all $P \in \partial \Omega$,

$$
\left\langle P, \sum_{i=1}^{N} K_{x_{i}(P)}\right\rangle=\sum_{i=1}^{N} P\left(x_{i}(P)\right)>0 .
$$

Applying Theorem 2.7 we get the existence of some $Q \in \Omega$ for which $\sum_{i=1}^{N} K_{x_{i}(Q)}=0$.

\section{REFERENCES}

[AdR88] J. Arias de Reyna, A generalized mean-value theorem, Monatsh. Math. 106 (1988), no. 2, 95-97. $\uparrow 2$

[BBC04] C. Bachoc, Ei. Bannai, and R. Coulangeon, Codes and designs in Grassmannian spaces, Discrete Math. 277 (2004), no. 1-3, 15-28. $\uparrow$

[BCN02] C. Bachoc, R. Coulangeon, and G. Nebe, Designs in Grassmannian spaces and lattices, J. Algebraic Combin. 16 (2002), no. 1, 5-19. $\uparrow 2$

[BB09] Ei. Bannai and Et. Bannai, A survey on spherical designs and algebraic combinatorics on spheres, European Journal of Combinatorics 30 (2009), no. 6, 1392-1425. Association Schemes: Ideas and Perspectives. $\uparrow 1$

[BD79] E. Bannai and R. M. Damerell, Tight spherical designs. I, J. Math. Soc. Japan 31 (1979), no. 1, 199-207. $\uparrow 4$

[BD80] _ Tight spherical designs. II, J. London Math. Soc. (2) 21 (1980), no. 1, 13-30. $\uparrow 4$

[BOC15] R. J. Berman and J. Ortega-Cerdà, Sampling of real multivariate polynomials and pluripotential theory, To appear in Amer. Jour. Math. Preprint arXiv:1509.00956 (2015). $\uparrow 2,6$

[BG15] J. S. Brauchart and P. J. Grabner, Distributing many points on spheres: Minimal energy and designs, Journal of Complexity 31 (2015), no. 3, 293-326. Oberwolfach 2013. $\uparrow 1$

[BEG16] A. Breger, M. Ehler, and M. Gräf, Quasi Monte Carlo integration and kernelbased function approximation on Grassmannians, Preprint arXiv:1605.09165 (2016). $\uparrow 2$

[BRV13] A. Bondarenko, D. Radchenko, and M. Viazovska, Optimal asymptotic bounds for spherical designs, Annals of mathematics 178 (2013), no. 2, 443-452. $\uparrow 1,2$, $3,4,6,8,10$

[BRV15] _ Well-Separated Spherical Designs, Constructive Approximation 41 (2015), no. 1, 93-112. $\uparrow 1,2$

[CC06] Y.Q. Chen and Y.J. Cho, Topological Degree Theory and Applications, Mathematical Analysis and Applications, CRC Press, 2006. $\uparrow 6$

[DX13] F. Dai and Y. Xu, Approximation theory and harmonic analysis on spheres and balls, Springer Monographs in Mathematics, Springer, New York, 2013. $\uparrow 4$

[dlHP05] P. de la Harpe and C. Pache, Cubature Formulas, Geometrical Designs, Reproducing Kernels, and Markov Operators (Laurent and Ceccherini-Silberstein Bartholdi Tullio and Smirnova-Nagnibeda, ed.), Birkhäuser Basel, Basel, 2005. $\uparrow 3$

[DGS77] P. Delsarte, J. M. Goethals, and J. J. Seidel, Spherical codes and designs, Geometriae Dedicata 6 (1977), no. 3, 363-388. $\uparrow 1$ 
[FM10] F. Filbir and H. N. Mhaskar, A quadrature formula for diffusion polynomials corresponding to a generalized heat kernel, J. Fourier Anal. Appl. 16 (2010), no. $5,629-657 . \uparrow 6$

[FM11] _ Marcinkiewicz-Zygmund measures on manifolds, J. Complexity 27 (2011), no. 6, 568-596. $\uparrow 6$

[Gau14] W. Gautschi, Walter Gautschi. Selected works with commentaries. Vol. 2, Contemporary Mathematicians, Birkhäuser/Springer, New York, 2014. Edited by Claude Brezinski and Ahmed Sameh. $\uparrow 1$

[GL15] G. Gigante and P. Leopardi, Diameter bounded equal measure partitions of Ahlfors regular metric measure spaces, arXiv:1510.05236 (2015). $\uparrow 2,5$

[HL08] K. Hesse and P. Leopardi, The Coulomb energy of spherical designs on $S^{2}$, Adv. Comput. Math. 28 (2008), no. 4, 331-354. $\uparrow 1$

[Hog82] S.G. Hoggar, t-Designs in Projective Spaces, European Journal of Combinatorics 3 (1982), no. 3, 233 - 254, DOI http://dx.doi.org/10.1016/S01956698(82)80035-8. $\uparrow 1$

[Kan15] D. M. Kane, Small designs for path-connected spaces and path-connected homogeneous spaces, Trans. Amer. Math. Soc. 367 (2015), no. 9, 6387-6414. $\uparrow 2$, 4

[KM93] J. Korevaar and J. L. H. Meyers, Spherical Faraday cage for the case of equal point charges and Chebyshev-type quadrature on the sphere, Integral Transform. Spec. Funct. 1 (1993), no. 2, 105-117. $\uparrow 2$

[KM94] _ Chebyshev-type quadrature on multidimensional domains, J. Approx. Theory 79 (1994), no. 1, 144-164. $\uparrow 2$

[Kui95] A. Kuijlaars, Chebyshev-type quadrature and partial sums of the exponential series, Math. Comp. 64 (1995), no. 209, 251-263. $\uparrow 4$

[LS04] Yu. I. Lyubich and O. A. Shatalova, Isometric embeddings of finite-dimensional $l_{p}$-spaces over the quaternions, Algebra i Analiz 16 (2004), no. 1, 15-32; English transl., St. Petersburg Math. J. 16 (2005), no. 1, 9-24. $\uparrow 1$

[MNW01] H. N. Mhaskar, F. J. Narcowich, and J. D. Ward, Spherical MarcinkiewiczZygmund inequalities and positive quadrature, Math. Comp. 70 (2001), no. 235, 1113-1130. $\uparrow 4$

[Neu81] A Neumaier, Combinatorial configurations in terms of distances, Technische Hogeschool Eindhoven (1981). $\uparrow 1$

[RSZ94] E. A. Rakhmanov, E. B. Saff, and Y. M. Zhou, Minimal discrete energy on the sphere, Math. Res. Lett. 1 (1994), no. 6, 647-662. $\uparrow 5$

[SZ84] P. D. Seymour and T. Zaslavsky, Averaging sets: a generalization of mean values and spherical designs, Adv. in Math. 52 (1984), no. 3, 213-240. $\uparrow 2,3$

[You01] R. M. Young, An introduction to nonharmonic Fourier series, 1st ed., Academic Press, Inc., San Diego, CA, 2001. $\uparrow 6$

Departamento de Matemáticas, Estadística y Computación, Universidad de Cantabria, Avd. Los Castros 44, 39005 Santander, Spain

E-mail address: etayomu@unican.es

Departament de Matemàtiques i Informàtica, Universitat de Barcelona \& Barcelona Graduate School of Mathematics, Gran Via 585, 08007, Barcelona, SPAIN

E-mail address: jmarzo@ub.edu

Departament de Matemàtiques i Informàtica, Universitat de Barcelona \& Barcelona Graduate School of Mathematics, Gran Via 585, 08007, Barcelona, SPAIN

E-mail address: jortega@ub.edu 